\title{
IMPLEMENTATION OF RAMP CONTROL IN RHIC *
}

\author{
Jörg Kewisch, BNL, USA
}

\begin{abstract}
After the injection of beam into RHIC the beam energy is ramped from $10.8 \mathrm{GeV} / \mathrm{u}$ to $108 \mathrm{GeV} / \mathrm{u}$ and the beta function of the interaction points is reduced from 10 meters to 1 meter. The set points for magnet power supplies and RF cavities is changed during such ramps in concert. A system of Wave Form Generators (WFGs), interconnected by a Real Time Data Link (RTDL) and and Event Link is used to control these devices.

RHIC ramps use a two level system of WFGs: onc transmits the beam energy and a "pseudo time" variable as functions of time via RTDL. the other calculates the device set points as functions of these RTDL variables. Energy scaling, saturation correction and the wiring of interaction region quadrupoles is performed on the second level.

This report describes the configuration and implementation of the software, firmware and hardware of the RHIC ramp system.
\end{abstract}

\section{INTRODUCTION}

The Relativistic Heavy Ion Collider (RHIC) consist of two intersecting storage rings. Using super-conducting magnets, RHIC will be able to collide ions from protons to gold. The existing AGS accelerator complex will be used as injector, supplying gold at $10.8 \mathrm{GeV} / \mathrm{u}$ and protons at $28.1 \mathrm{GeV}$. The beams are then accelerated in RHIC to 108 $\mathrm{GeV} / \mathrm{u}$ and $249 \mathrm{Gev}$, respectively. All ion beams except protons will cross the transition energy during acceleration. At storage energy, the beta functions in the interaction points are reduced from 10 meters to 1 meter.

The RHIC ramp system, which brings the beams from injection to storage, controls the currents of the magnet power supplies including special jump power supplies for the transition crossing, cavity voltages and parameters for the RF phase and frequency feedback systems.

This system must fulfill the following requirements:

1) The system must be programmable to execute a complete ramp sequence on a start signal. This is especially important since the emittance of a gold ion beam grows rapidly at injection energies due to intra beam scattering. The complete sequence includes an acceleration ramp with transition crossing and the beta squeeze ramp. It is important that these ramps can be executed without delays once the machine is filled.

2) The system must allow tweaking of some components during and after the programmed ramps. Due to the large inductance of the super-conducting magnets the ramp speed is slow. A typical acceleration ramp takes $90 \mathrm{sec}-$ onds. It is desirable that the operator has the ability to influ-

\footnotetext{
- Work performed under the auspices of the US Department of Energy.
}

ence parameters like tunes, chromaticities and orbit bumps after a ramp is started. After the completion of a ramp the beam life time may be optimized by tweaking. Tweaking must be possible without major effort.

3) The system must support the easy control of magnet currents which are not supplied by a single power supply but are generated by a main power supply and several trim power supplies. The interaction region quadrupoles are wired this way in order to minimize the number of warmcold feed-throughs.

4) The system must provide constant monitoring of magnet and RF parameters as well as a possibility for post mortem analysis after a magnet quench.

\section{CONFIGURATION OF THE SYSTEM}

The design of the RHIC controls hardware was inspired by the Fermilab control system. The general tool for the ramp control is the "Wave Form Generator" (WFG). The WFG is a VME module containing a computer based on the INTEL i960 CPU. Each WFG module has two fiber optics outputs which can be used in two ways: as the current reference for a power supply or as a source of the Real Time Data Link (RTDL).

RTDL [2] is one of two dedicated data lines around the ring. It transmits 256 "frames" with a update frequency of $720 \mathrm{~Hz}$. Each frame contains a 24 bit integer number which can be generated by software, a WFG or any other device with a fiber optics output. The frames can be read by WFGs and used to determine the next output values.

The other data line is the event link [3]. This line provides 256 different start signals (events) to the hardware. This link is used to start ramps or change operation modes for WFGs, stop data collection into circular buffers after a quench, trigger the current jump of the $\gamma_{\iota}$ quadrupoles and many other applications outside the ramp system.

The "Multiplexed Analog to Digital Converter" (MADC) [4] is used for monitoring the system. Each MADC module can monitor 64 signals and is programmable to scan one channel up to $50 \mathrm{kHz}$ or all channels with a lower rate.

A PLC system allows switching power supplies on and off and reports error conditions of the power supply (regulation error, etc) and magnet (quench).

WFGs are programmed by specifying up to 15 "formulas". Events from the event link are used to switch from one formula to the next. Formulas may contain the following elements:

1. add, multiply and shift operations.

2. linear and cubic spline lookup in programmable tables. 
3. Function of time: $F(t)$ is given in a table with linear interpolation.

\section{RTDL frames and programmable constants.}

All operations are performed using 32-bit integers, floating point operations are not available. However, a "multiply and shift" operation with an intermediate 64-bit product is available.

The WFGs are used in a two level configuration (Figure 1): A pair of WFGs on the first level generate the beam rigidity $B \rho$ and the "pseudo time" $P$ as functions of time and distribute the value via RTDL. $P$ is a function calculated to limit the second derivative of the magnet current $\ddot{I}(t)$ when $I(P)$ is a cubic function of $P$. In addition, RTDL is used to transmit the values of "knobs" used for tweaking.

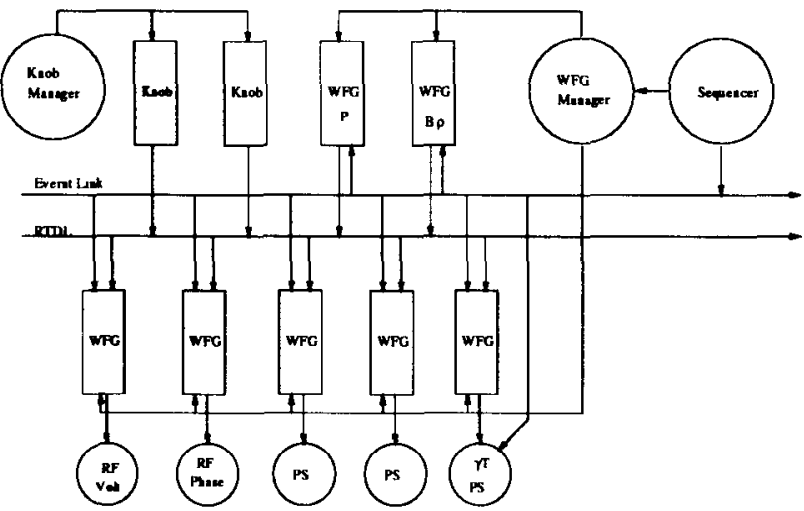

Figure 1: Two level WFG configuration using RTDL and Event link.

On the second level are WFGs which calculate magnet currents and RF parameters.

$$
\begin{aligned}
k= & F_{\text {ideal }}(P)+F_{\text {trim }}(P) \\
& +\left[c_{1} \cdot k_{1}\left[+c_{2} \cdot k_{2}\right]\right] \\
B= & k \cdot B \rho \\
I_{\text {magnet }}= & F_{\text {fielddat } a}(B)
\end{aligned}
$$

$k$ is the magnet strength (deflection angle for dipoles, inverse focal length for quadrupoles, etc.) which is the sum of the programmed strength given as functions ${ }^{1}$ of $P$ and optional knob values. $k_{1}$ and $k_{2}$ are RTDL knob frames, $c_{1}$ and $c_{2}$ are the conversion constants from knob value (i.e. tunes or orbit displacement) to magnet strength.

From the total value of $k$ the magnetic field or gradient $B$ is calculated and, using the measured field data, the magnet current $I_{\text {magnet }}$.

The thing left to do is to calculate the power supply current. Figure 2 shows the wiring of the interaction rcgion quadrupoles. Each of the power supplies generates the difference of two magnet currents. Since the WFGs do not

\footnotetext{
${ }^{\prime} F_{t \text { deal }}(P)$ contains the settings for the ideal machine, $F_{t r i m}(P)$ contains corrections
}

communicate with each other ${ }^{2}$, this functionality was implemented in the power supply interface card (Figure 3).

The power supply interface card has two WFG inputs and calculates the difference value. Of course both signals must have the same scale factor which was chosen to be $1 \mathrm{~mA}$ per bit. The result is then multiplied, divided and shifted to produce a 16 bit number which maximum value corresponds to the maximum current of the power supply. A DAC converts this number into the reference voltages for the power supply.

Figure 3 shows also the signals monitored by the MADC: The reference current (set point), the measured current, the error current ${ }^{3}$ and the voltage. ${ }^{4}$ These signals are sampled with $60 \mathrm{~Hz}$ and stored in a circular buffer. Sampling is stopped by the "quench event" which is generated by the quench protection system. The data buffers are inspected for the post-mortem analysis before the system is restarted.

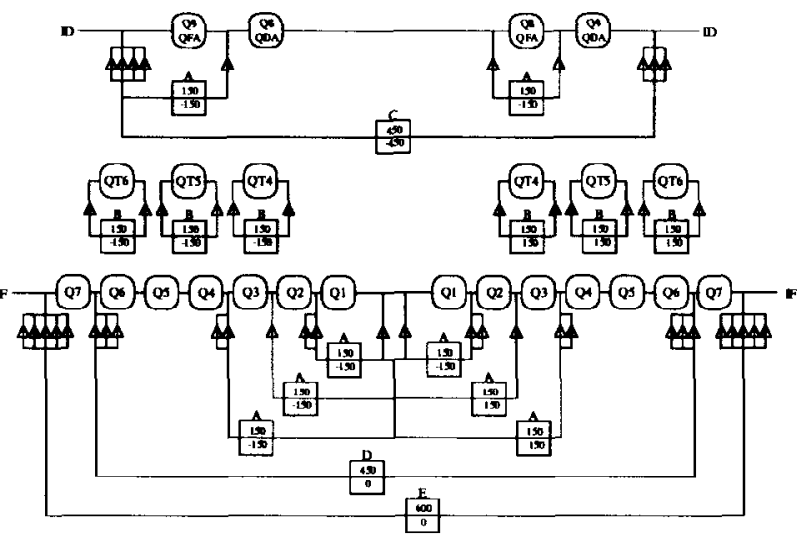

Figure 2: Power supply configuration for the IR quadrupoles.

\section{SOFTWARE}

WFGs, MADCs and PLCs are located in VME crates which are distributed around the ring. Each crate has a "Front End Computer" (FEC) which th a tcp/ip connection to the "Console Level Computers" (CLC). FECs allow access to WFGs, PLCs and MADCs through the "Accelerator Device Object" (ADO) software. ADOs provide a high level interface to accelerator devices and hide hardware details from the outside world. However, they control only a single device.

"Manager" programs run on the CLC level and provide the controls of logical groups of devices. The "WFG Manager" is the program that loads all ramp data into the WFGs. It can load several ramps using different formu-

\footnotetext{
${ }^{2}$ The current set points of the main power supplies are available as RTDL frames and are subtracted in the WFG.

${ }^{3}$ The error current is the difference of reference current and measured current, multiplied by $\mathbf{5 0}$ for better resolution.

${ }^{4}$ The main power supplies for dipoles and quadrupoles have additional signals for quench detection.
} 


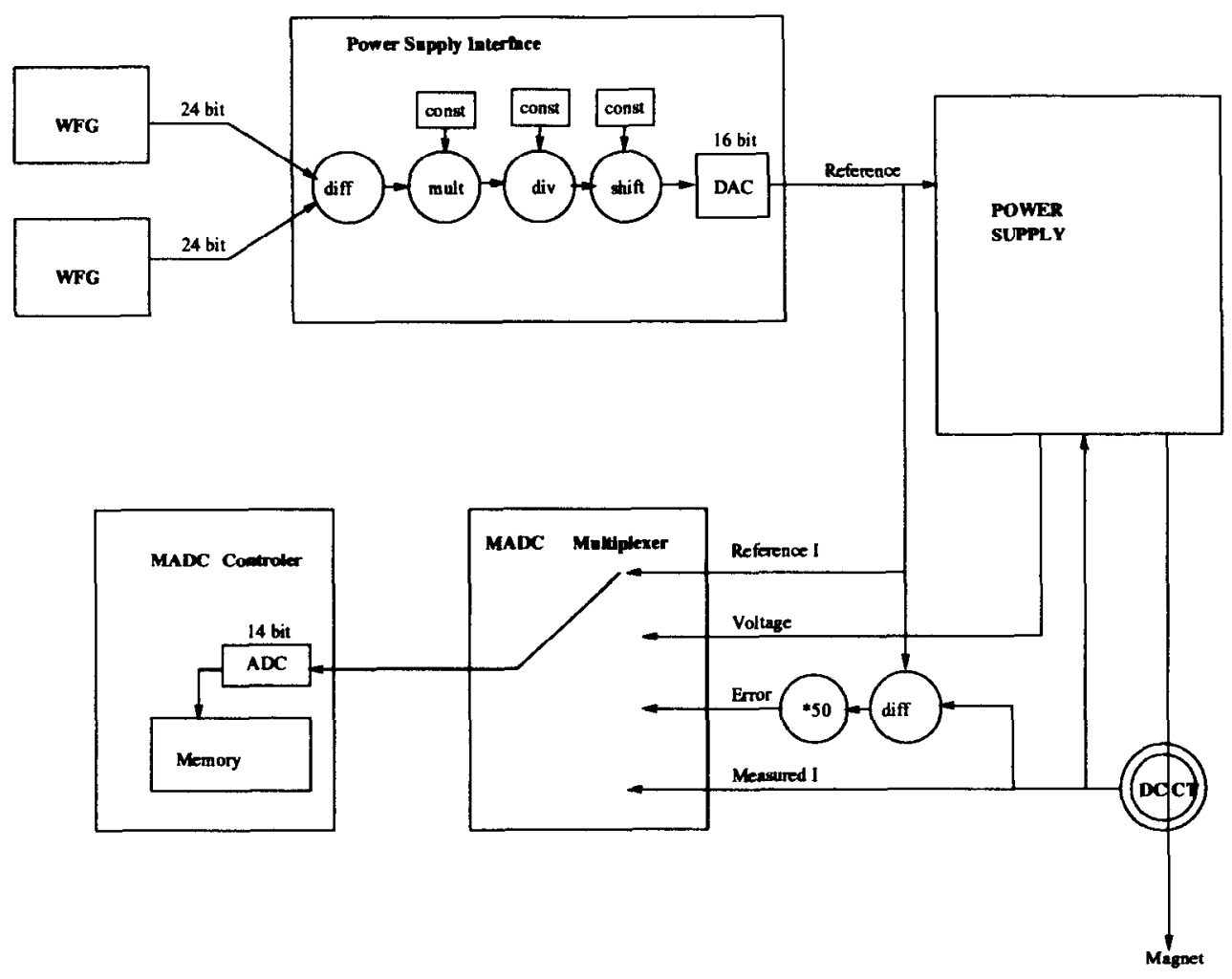

Figure 3: Control and monitoring of a power supply.

las and tables. Each ramp is connected to a specific event. The "Sequencer" is a scripting tool which is used to trigger these events and, ultimately, orchestrate to the whole injection-acceleration-storage cycle. More detail is given in [8].

\section{CONCLUSION}

The Ramp Control System for RHIC is build on powerful hardware which allows ramps to be programmed in advance and executed without operator intervention. However, tweaking of parameters during a ramp, which may take several minutes, is not excluded. This provides a valuable tool during commissioning.

The system is designed from the accelerator physicist point of view. Set points are integrated magnet strength and high level parameters and are converted into currents and voltages close to the hardware level. This allows encapsulation of hardware details and provides a simple interface for application programs.

The MADC system provides powerful post-mortem analysis.

\section{REFERENCES}

[1] T. Clifford, V. Mane, S. Peggs, "RHIC Ramps: a proposal", RHIC/AP/16, December 1993.

[2] H. Hartmann, "Specifications for the RHIC Real Time Data Link transmitter system", October 1993.

[3] H. Hartmann, B. Oerter, S. Peggs, "RHIC Real Time Data Link System", October 1993.
[4] R. Michnoff, "The RHIC General Purpose Multiplexed Analog to Digital Converter System", PAC 1995.

[5] T. Kahn, "RHIC Power Supply Waveform Generator Board Application Notes", revised October 1994.

[6] T. Kahn, "Power Supply Waveform Generator Programmer Manual", March 1995. October, 1994.

[7] J. Kewisch, V. Mane, T. Clifford, H. Hartmann, T. Kahn, B. Oerter, S. Peggs, Model for RHIC Ramp Controls, EPAC 1996.

[8] J. Kewisch, J. van Zeijts, S. Peggs, T. Satogata, "Ramp Management in RHIC", these proceedings. 